\title{
Comparing Isometric and Elastic Surfboard Interfaces for Leaning-Based Travel in 3D Virtual Environments
}

\author{
Jia Wang ${ }^{1}$ \\ ${ }^{1}$ HIVE Lab \\ Worcester Polytechnic Institute
}

\author{
Robert W. Lindeman ${ }^{1,2}$ \\ ${ }^{2}$ HIT Lab NZ \\ University of Canterbury
}

\begin{abstract}
Inspired by the Silver Surfer comics we developed a leaningbased surfboard interface which allows the user to fly in 3D virtual environments by shifting his/her center of mass on the board. The interface works in either an elastic tilt mode or an isometric balance mode offering different equilibrioceptive feedback to the user. Interested in how different levels of equilibrioceptive feedback influence the usability of this interface, a formal user study was conducted comparing the two modes in both separated and combined pitch and yaw travel tasks. Six of 30 subjects dropped out of the experiment because of cyber-sickness and were interviewed by the experimenter. Statistical analysis of data from the remaining subjects showed that although objectively there was no significant difference between the two modes regarding training effects and task performance, subjectively most subjects preferred the elastic tilt mode because of its intuitiveness, realism, fun, and sense of presence despite the greater fatigue and after effects (e.g., loss of balance) of using it. Based on the results we suggest a general preference of elastic devices and several design guidelines to future $3 \mathrm{D} \mathrm{VE}$ travel interface designers.
\end{abstract}

KeYwords: Isometric and elastic, 3D travel interface, leaningbased interface, board interface

IndeX Terms: I.3.6 [Computer Graphics]: Methodology and Techniques - Interaction techniques; I.3.7 [Computer Graphics]: Three-Dimensional Graphics and Realism - Virtual reality.

\section{INTRODUCTION}

In virtual reality (VR), travel refers to the motor process of changing the position and orientation of one's virtual viewpoint to navigate from point $\mathrm{A}$ to $\mathrm{B}$ in an immersive virtual environment (VE). Designing travel interfaces has been a challenging problem because of the requirement of realistically, efficiently, and precisely mapping the user's limited locomotion in a finite realworld space to that of a potentially infinite virtual space. In addition, the usability of a travel interface impacts not only the efficiency of performing travel tasks, but also physiological and psychological human factors such as cyber-sickness [16], spatial orientation [17], and virtual-world cognition [24].

Recent locomotion research has focused on realizing endless real walking in a limited lab space either by building sophisticated mechanical systems that repeatedly place floor tiles on predicted positions and heights of the user's steps [6][7][8] or by gradually

${ }^{1}$ WPI CS Dept., 100 Institute Road, Worcester, MA, 01609, USA

${ }^{2}$ Private Bag 4800, Christchurch, 8140, New Zealand

\{wangjia,gogo\}@cs.wpi.edu

IEEE Symposium on 3D User Interfaces 2012

4-5 March, Orange County, CA, USA

978-1-4673-1205-9/12/\$31.00 @2012 IEEE rotating the virtual world to redirect the user to walk in circles in the real world. Because of its success in preserving proprioception [11], comparative experiments have shown that when used as a travel interface, real walking has significant advantages in traveltask performance, VE immersion and cognition compared to joysticks [24]. Yet, this is not a consensus and other researchers believe that a well-designed joystick travel interface can achieve the same level of efficiency at a much lower cost [15].

In our current work, we are interested in 3D VE travel in which the user's avatar is no longer restricted to terrain, but is allowed to fly in any direction. 3D VE travel interfaces are usually seen in multi-modal and multi-sensorial entertainment systems but can also be used in virtual cultural heritage modeling, virtual data visualization, and virtual tourism where the ability to move in three dimensions is beneficial. Because the interfaces discussed previously are mainly designed for $2 \mathrm{D}$ terrestrial walking, it may not be easy to expand them to 3D VE travel while maintaining the same efficacy. Meanwhile, inspired by the success of personal transporters in real life, such as the Segway Patroller and the Honda Personal Hovercraft, several leaning-based travel VR interfaces have been proposed. They allow the user to control the travel direction and speed by shifting his/her center of gravity while standing on the floor [9] or a platform [10][21] or sitting in a chair [1]. Unlike walking interfaces that try to simulate and preserve users' proprioception, leaning-based interfaces emphasize preserving realistic equilibrioception [10] to increase immersion. There has not been formal experiments comparing walking interfaces with leaning-based interfaces for 2D terrestrial travel tasks, but preliminary evaluations of the latter have shown promising prospects with a much lower cost and smaller space requirement. Furthermore, because leaning-based interfaces occupy an intermediary position between "real life simulation" walking interfaces and "virtual world abstraction" joystick interfaces, they may lead to high levels of both immersion and efficiency when used as 3D VE travel interfaces.

In the following sections, we present a leaning-based surfboard interface inspired by the Silver Surfer comics. By balancing his/her center of mass on the board to control the travel direction and raising one of his/her arms to control the travel speed, the user is able to fly any distance and in any direction in a 3D VE. The interface is able to work either as an isometric balance board (IBB) or an elastic tilt board (ETB), providing different levels of equilibrioceptive feedback. Our main objective was to compare these two modes in a multi-sensorial VR system of fulfilling target search and collection tasks to investigate how different levels of equilibrioceptive feedback influence the quantitative and qualitative usability of such leaning-based 3D travel interfaces.

\section{Related Work}

\subsection{Travel Interfaces}

To achieve the goal of providing users with realistic sensations of travel experience while keeping them in a limited real space, 
many input devices have been proposed and evaluated. Classical controllers such as mice, keyboards, joysticks, and game pads were the first to be considered because of their low cost and success in video games. Although these devices are capable of easily displacing the viewport, the lack of proprioceptive and vestibular feedback when travelling leads to a poor sensation of locomotion in the VE [3]. To make virtual travel more intuitive, several researchers tried to bring real walking into the limited lab space by developing different types of platforms or mounting orientation and acceleration sensors on the user's body.

Based on the idea of mechanically bringing the floor tiles left behind by the user to the front where his/her feet are going to step next, numerous sophisticated hardware systems were proposed and developed. Iwata et al. built the Omni-directional Treadmill [8] and the CirculaFloor [6] locomotion interface which support endless travel in all directions on a 2D surface, as well as the Gaitmaster interface [7] which allows uni-directional travel on uneven terrains. However, all these systems require the user to travel very slowly and carefully because of the great time and effort to mechanically displace the floor tiles, and the safety risk involved in the process.

Templeman et al. [19] designed and implemented the Gaiter system for walking-in-place (WIP) travel. Multiple acceleration, orientation, and pressure sensors were mounted on special locations of the user's body to track gestures of in-place turning, stepping, and strafing. The system included a torso-mounted framework dropping from the ceiling to hold the user in a small area. Backward walking was implemented by an additional gesture because of the difficulty to differentiate forward and backward WIP gestures by the sensor data alone. By designing the gestures to mimic real walking, the WIP interface offers high proprioceptive nevertheless insufficient vestibular feedback.

The HiBall tracker developed by 3rdTech, based on an earlier project at the University of North Carolina at Chapel Hill, allows position and orientation tracking in a relatively large range. Based on this technology, a real walking interface was proposed in which the user wears a tracker and naturally walks in a large lab space to travel in a VE of the same size. The researchers compared this technique with WIP and joystick flying and reported significantly higher sense of presence for the real walking technique [20]. Other research work has tried different approaches to take real walking further to realize infinite virtual world travel. The Magic Barrier Tape relies on pushing "do not cross" tape to map the same limited real space to dynamic equivalently sized regions of the virtual space [4], but breaks the user's immersion when the mapping changes. Razzaque et al. [14] presented an innovative redirected walking technique based on observations from a field study that fully immersed users (no vision from outside world) do not actually walk in straight lines when they believe they were doing so, and it is hard for them to notice small gradual virtual world rotations when they are walking. Based on these facts the researchers imperceptibly rotate the virtual world little by little when the user is walking and by larger amounts when the user's head is rotating, and are able to redirect the user to walk in circles within a limited lab space without breaking the immersion. Follow-on work introduced specifically designed distracters in the VE based on predictions of the user's future locomotion path to lower the possibility of the user discovering the underlying virtual world rotations [12]. However, such techniques still cannot handle special conditions such as frequent, fast, and sharp turns.

On the other hand, effort has also been put into converting successful real life personal transporters to VR travel interfaces. Valcov et al. implemented the virtual Segway Patroller travel interface using the Wii Fit Balance Board, which is an IBB [21]. The user stands on the board facing forward, and controls his/her travel speed by leaning forward or backward and his/her travel direction by leaning to his/her left or right. The Joyman interface developed by Marchal et al. treats the user leaning on a tilt platform as a human-scale joystick, and maps the tilt data according to a pre-defined model to control virtual locomotion [10]. Compared to real walking interfaces, leaning-based interfaces are easier to be adapted to $3 \mathrm{D}$ VE travel scenarios in which at least three degrees of freedom (DOF) are needed. Valcov et al. programmed the IBB to identify a special foot gesture when the user leans one foot on its toe and the other on its heel. Depending on how much they differ from each other, the avatar's position changes along the up-axis at different rates. Nevertheless, this approach is not very intuitive and effective, and is prone to unintentional input caused by the user trying to maintain his/her balance. Wang and Lindeman [23] proposed a multi-modal VR system which included a board interface specifically designed for $3 \mathrm{D}$ VE travel. However no experiment was done to prove the benefit of using such interfaces. The surfboard interface presented in this paper is similar to these interfaces but is specifically designed to function either as an IBB or an ETB, with a focus of investigating how different levels of equilibrioceptive feedback impact the usability of leaning-based interfaces when used to fulfill 3D VE travel tasks.

\subsection{Isometric, Isotonic, and Elastic Interfaces}

The terms isometric and isotonic come from exercise physiology. An isometric contraction happens when there is tension on the muscle but no movement is made, causing the length of the muscle to remain the same. On the other hand, in isotonic contraction, tension remains unchanged but the muscle's length changes. In the context of human-computer interaction, according to Zhai [25], an isometric device is one that senses force but does not perceptibly move, such as the IBB, while an isotonic device has zero or negligible resistance, but senses its own movement, such as with mice. Between the isometric and the isotonic, elastic devices refer to those whose resistive forces increase with displacement, such as traditional joysticks, or the Joyman interface [10].

In 1993, Zhai proposed a scheme to comparatively investigate isometric, isotonic, and elastic devices for either rate-controlled or position-controlled 6-DOF object manipulation tasks [27]. Based on the scheme, the first experiment showed that by using isometric rate control and isotonic position control, subjects spent less time to complete the tasks than using other combinations [27]. The second experiment showed that in the rate-controlled scenario, a hand-held elastic device had superior performance over the isometric one but such superiority was no longer evident after 20 minutes of practice [26]. We have adapted Zhai's results for use with locomotion interfaces, and conducted experiments about their utility.

\section{Methodology}

\subsection{The Surfboard Travel Interface}

General 3D space navigation consists of six DOFs in two categories: pitch, roll, and yaw for orientation control, and translations along the $\mathrm{X}, \mathrm{Y}$, and $\mathrm{Z}$ axes for location control. The fictional "Silver Surfer" can pitch, roll, and yaw his surfboard as well as speed up and move forward, giving him control of 4-DOF locomotion by which he can travel to anywhere in the space. Because in essence three DOFs are sufficient to completely travel in 3D, and according to Vidal and Amorim [22], roll (rotation around the forward direction) goes against the human natural balance system and may lead to severe cyber-sickness and spatial disorientation, only the control of pitch, yaw, and forward 
translation of the virtual board were realized in our system, as illustrated in Figure 1.

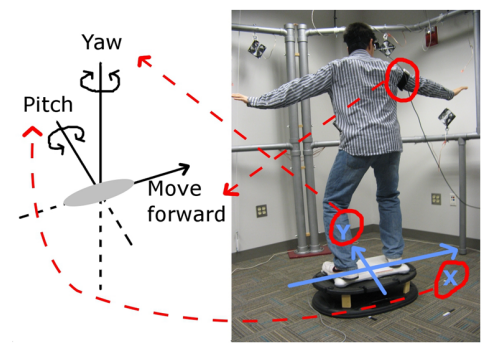

Figure 1. The three DOFs implemented by the travel interface

\subsubsection{Speed Control}

The first DOF, the control of the travel speed along the forward direction, was realized by mounting a Wireless-T B-Pack Compact Wireless Accelerometer (model WAA-001) on the user's forward arm, i.e., the left arm for a normal surfer (left foot forward) and the right arm for a goofy surfer (right foot forward). The arm orientation was mapped to the virtual travel speed by an adapted Go-Go technique [13], so that the user was able to precisely explore a small local area, as well as efficiently travel over long distances.

$$
\begin{cases}s=a x, & \text { if } 0 \leq x \leq D \\ s=a x+\frac{(x-D)^{2}(M-a)}{(1-D)^{2}}, & \text { if } D \leq x \leq 1\end{cases}
$$

Equation 1 shows the formula of the speed control law. $S$ represents the travel speed in the VE. Variable $x$ is the arm lift angle normalized and clamped to $[0.0,1.0]$, with the arm completely relaxed by the waist being 0.0 and it raised to 90 degrees or higher being 1.0. The constant $D$ is the boundary point from linear to non-linear mapping. By iterative user tests, its value was empirically set to 0.4 to place the linear-to-exponential transition point around an arm lift of 35 degrees. When $x$ is smaller than $D$, it is scaled linearly by the constant $a$ (empirically set to 133.3 meters per second - two-thirds of the maximum speed) to control the speed. And when $x$ is larger than $D$, the second equation maps it non-linearly to cover a greater speed spectrum. The constant $M$ is equal to the maximum travel speed (set to 200 meters per second for our VE), which is used in the formula to regulate the speed growth so that when $x$ increases from $D$ to 1.0 (arm fully raised), the speed increases smoothly at the beginning and drastically until reaching the maximum speed.

Although alternatively the arm orientation could has been used to fly up and down while leaning on the board controlled speed, preliminary user tests showed that this was not intuitive for a surfboard metaphor and was therefore not used in our design.

\subsubsection{Direction Control}

The control of the other two DOFs, namely, the pitch and yaw of the virtual board, was supported by the surfboard interface. A Wii Fit Balance Board was used as the IBB and a Reebok Core Trainer Tilt Board was used as the ETB. The former is a sturdy plastic panel that rests on four feet, each containing a pressure sensor that streams pressure values to the computer via Bluetooth $^{\mathrm{TM}}$ which can be synthesized to obtain the user's center of gravity as a pair of values [5]. The latter is a fitness board which tilts in all directions. The rubber mountings in it resist tilt to keep the top surface parallel to the ground, offering elastic force feedback to the user. Both devices provide 2-DOF data (pitch and roll of the ETB and the user's center of mass on the IBB) that can be mapped to the pitch and yaw of the virtual board. In our system, the IBB was attached on top of the ETB using industrialstrength Velcro hook and loop fasteners. The integrated interface switches between ETB mode and IBB mode by adding/removing a supportive piece of wood on each of the four corners below the tilt board as shown in Figure 2. The wood pieces are inserted for IBB mode and removed for ETB mode. The pressure sensor data of the IBB is used to track the user's center of gravity in both modes. The integration of the two boards also removed potential biases against direct comparison because of the height and surface size differences between the two devices.

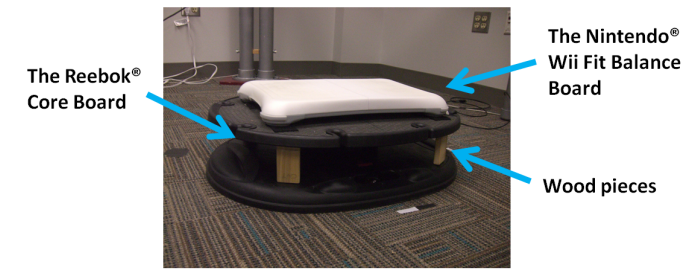

Figure 2. The integrated interface switches between ETB and IBB mode by removing and inserting wood pieces

When standing on the board, different users may shift their center of mass over different ranges because of differences in weight, height, and balance skills. Therefore, a calibration procedure was necessary to ensure the same travel potentiality for every user. To calibrate the board, the user was asked to lean as much as he/she could in each direction (forward, backward, left and right) following the pictorial instructions in Figure 3, and the sensor data was recorded as normalization dividends. This process was executed twice by each user to calibrate the two modes separately because the range of center of gravity is smaller in ETB mode due to the resistive force feedback.

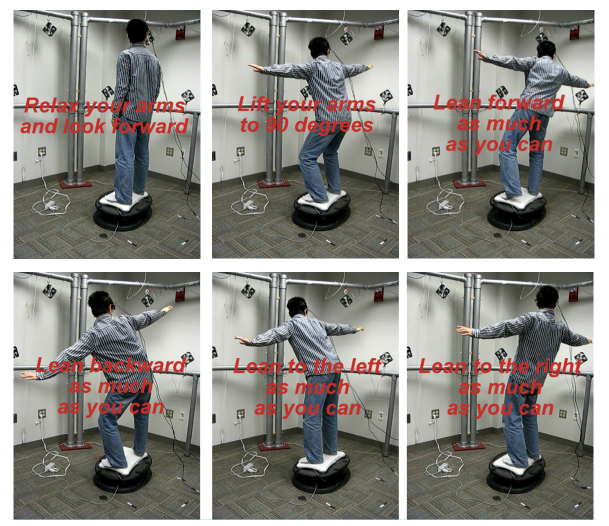

Figure 3. The user follows these pictorial instructions to calibrate the board in both modes (ETB mode shown in this figure)

Position control and rate control are two different approaches to map data from the devices to values of variables in the virtual world [27]. Using the former, the device controls the exact value of the variable directly while with the latter it controls how fast the value changes. Both position control and rate control can be applied to map the data from the physical board to pitch and yaw of the virtual board. However, the sensor range, the deviation of the center of gravity from the board center, is much smaller than the virtual-board range, the \pm 180 degrees of pitch and yaw. Therefore, it may be optimal to clamp the latter. For example, the pitch of the virtual board needs to be clamped to not exceed \pm 90 degrees, otherwise the user will be able to turn his/her virtual viewport upside down while his/her real body is still upright, in which situation the user will get confused, disoriented, or even nauseated because the vision of the virtual world would drastically contradict what his/her vestibular system perceived 
from the real world [22]. In a pilot study that consisted of 10 lab members, the original and clamped position and rate control were all tested and the feedback from the subjects are summarized in Table 1. The clamped position control and unclamped rate control were chosen for pitch and yaw mapping respectively so that the user can easily adapt to the interface and use it to travel completely in three dimensions.

Table 1. The result of the pilot study evaluating different control laws for pitch and yaw control of the virtual board

\begin{tabular}{|c|c|c|}
\hline Mapping DOF & Pitch & Yaw \\
\hline $\begin{array}{c}\text { Position } \\
\text { control }\end{array}$ & $\begin{array}{c}\text { Upside down vision } \\
\text { nauseates and disorients } \\
\text { the user }\end{array}$ & $\begin{array}{c}\text { Small input range to } \\
\text { large output range }\end{array}$ \\
\hline $\begin{array}{c}\text { Position } \\
\text { control } \\
\text { (clamped) }\end{array}$ & Good & $\begin{array}{c}\text { Unable to travel in } \\
\text { all directions }\end{array}$ \\
\hline $\begin{array}{c}\text { Rate } \\
\text { control }\end{array}$ & $\begin{array}{c}\text { Upside down vision } \\
\text { nauseates and disorients } \\
\text { the user }\end{array}$ & Good \\
\hline $\begin{array}{c}\text { Rate } \\
\text { control } \\
\text { (clamped) }\end{array}$ & $\begin{array}{c}\text { Unintuitive and hard to } \\
\text { understand }\end{array}$ & $\begin{array}{c}\text { Unable to travel in } \\
\text { all directions }\end{array}$ \\
\hline
\end{tabular}

Figure 4 illustrates how the sensor data was processed. The raw center of gravity data from the IBB sensors was first normalized by the calibrated ranges to calculate how much the user shifted his/her center of gravity off the neutral position around both axes of his/her maximum. These percentages were then linearly mapped to the pitch angle range ( \pm 50 degrees) for clamped position control and to the yaw speed range ( \pm 60 degrees per second) for unclamped rate control to update the spatial orientation (i.e., the travel direction) of the virtual board. The aforementioned Go-Go technique was also tested in the pilot study in place of the linear mapping for both position controlled and rate controlled yaw, which nevertheless was not favored by the subjects because the board's small input range and the difficulty to precisely adjust one's balance made the transition from linear to non-linear mapping (when $x$ is around $D$ in Equation 1) too steep to control.

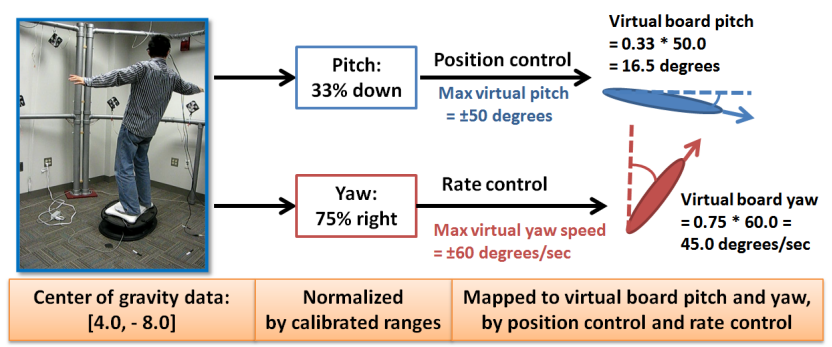

Figure 4. The raw data from the sensor get normalized and linearly mapped to control the pitch and yaw of the virtual board

\subsubsection{Device-directed Travel Interface}

Bowman et al. [2] categorized travel interfaces into gaze-directed (moving in the direction the user is looking), pointing-directed (moving in the direction the user is pointing), and torso-directed (moving in the direction the user's torso is facing). Our space surfing metaphor belongs to a fourth category, namely devicedirected interfaces, because from the immersed user's perspective, the virtual board, like a vehicle, always moves towards the same direction the real board is facing, no matter where the user is looking, pointing, or facing. In other words, when the user is granted the ability to look around the virtual world by turning his/her head, it is possible for him/her to fly in one direction while looking in another. In our system, we use a Space Point Fusion sensor to track the user's head orientation and render visuals accordingly. A good analogy to explain the relationship between the orientation of the virtual board and that of the virtual camera is when a person looks around on a swiveling chair. The viewing direction of the person is decided both by the orientation of his/her head and that of his/her body which is coupled with the chair. To keep the focus on an external object that is not rotating with the chair, the person has to keep turning his/her neck to compensate for the rotation of the chair. This is similar to what happens to the immersed user in the VE. In order to travel to an object, the user needs to turn the virtual board towards it and adjust his/her head simultaneously to compensate the focus deviation caused by the board rotation. The only problem is that because the real board is not actually turning, the absence of vestibular feedback may require extra thinking of the user to figure out the right actions.

The problem was evident in a public demonstration when users were surfing on the ETB alone (without the IBB attached on top but tracked by an accelerometer) that has a surface big enough for them to unconsciously shuffle their feet and turn their bodies. Without real world vision they were not able to reorient to the right stance, and finally lost the control of 3D travel because they were not able to identify the front side of the real board which maps to the forward direction of the virtual board.

\subsection{The Virtual Environment}

The VE was developed using the Unity3D game engine with all input devices driven by the Virtual Reality Peripheral Network (VRPN) [18] and the Unity Indie VRPN Adapter (UIVA). As shown in Figure 5, an infinite virtual world is created by repeating nine identical large terrain tiles in eight geographical directions based on the current location of the virtual avatar. The avatar stands on a virtual board whose speed and direction are controlled by the travel interface, either in ETB mode or IBB mode. Clouds, trees and various terrain types such as mountains, valleys and plains are included to increase realism and provide motion cues. Canister targets are distributed in the sky for the user to collect.

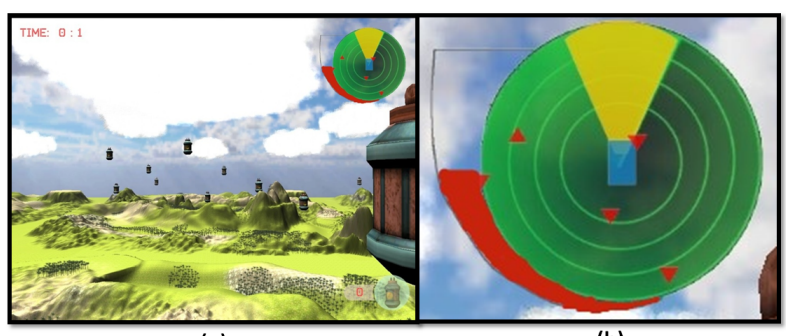

(a)

(b)

Figure 5. (a) The infinite virtual world; (b) the forward-up radar helps the user with way-finding

To decrease the demands of way-finding and focus the experiment mainly on travel, a forward-up radar is rendered as a graphical user interface (GUI) component in the top right corner of the user's view, which is magnified in Figure 5(b). The red triangles indicate the canisters' locations relative to the virtual board, with the point of a triangle used to indicate height (above/below the board). The virtual board is represented as a blue rectangle in the middle which always faces forward on the radar. The yellow sector corresponds to the user's viewport and the red bar to the left of the radar indicates the user's current surfing speed. Other GUI components include a timer in the left top corner which shows how long the user has been immersed in 
the VE, and a counter next to the canister icon in the right bottom corner showing how many targets have been collected so far.

All graphical contents are rendered to the two screens of an eMagin z800 HMD in non-stereoscopic mode. The stereoscopic module of the HMD was turned off because the user looks at faraway objects in the virtual world most of the time which have little binocular parallax. To provide tactile feedback, the TactaCage system was used to simulate wind. This system was designed for an immersed user to stand in the middle and feel directional or field wind from computer controlled fans mounted around the perimeter of the framework, as shown in Figure 6. To provide a more realistic sensation of the surfing speed, seven muffin fans in front of the surfboard were used whose speeds were directly mapped from the virtual surfing speed, which was in turn controlled by the arm-mounted accelerometer. To prevent the users being distracted by the noise of the fans, they wore a noiseproof headset which repeatedly played elevator music.

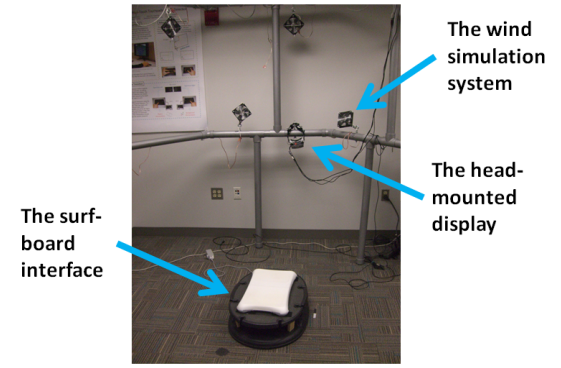

Figure 6. The TactaCage system provides wind tactile feedback

\section{EXPERIMENT}

\subsection{User Study}

The formal user study was designed to inspect pitch and yaw both separately and integrally, as shown in Figure 7, to reduce the chance of important patterns being hidden in the data resulting from our experiments. In the pitch and yaw sub-experiments, the yaw and the pitch of the virtual board were disabled respectively to make the virtual travel two dimensional. The subject could look in any direction by turning his/her head and control the travel speed by raising and relaxing his/her tracked arm. In the combined sub-experiment, the subject was able to pitch and yaw the virtual board simultaneously giving him/her the ability to completely navigate the VE in 3D.

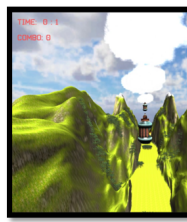

(a)

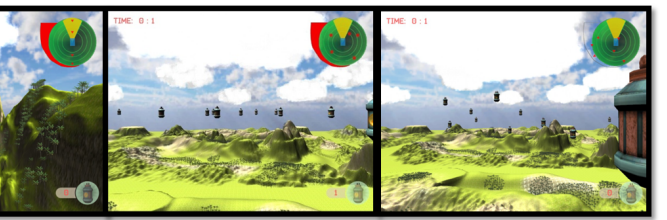

(b) (c)
Figure 7. (a) The pitch level only allows the user to pitch up/down.

(b) the yaw level only allows the user to yaw left/right. (c) the combined level allows the user to both pitch and yaw.

Table 2 summaries the user study design. The board mode is varied in each experiment creating six conditions. Each condition consisted of a training session and a study session. The study was conducted within subjects. Each subject went through the pitch experiment, the yaw experiment, and the combined experiment in sequence. The order of the interface (IBB and ETB) was kept the same within each subject for the three experiments, but was randomized between different subjects.
Table 2. Formal User Study Design

\begin{tabular}{|c|c|c|c|}
\hline Board & Pitch & Yaw & Combined \\
\hline ETB & ETB-Pitch & ETB-Yaw & ETB-Combined \\
\hline IBB & IBB-Pitch & IBB-Yaw & IBB-Combined \\
\hline
\end{tabular}

When a subject arrived, he/she was first asked to fill in a general information form which included demographic questions such as gender, age, height, weight, surfing stance (goofy or regular), Wii Fit Balance Board experience, fitness center practice board experience, real life board surfing experience, surfing-type video game experience and a self-evaluation of balance skills from 0 ("cannot balance at all") to 10 ("can balance very well"). After that, he/she calibrated the board in both modes and went through all six conditions. The training session in each condition was designed as a test to ensure a minimum level of proficiency. In order to pass the test and start the corresponding study trial, the subject had to collect a certain number of targets with the last two consecutive targets collected within a certain amount of time. The specific requirements were empirically set to $20 / 10 / 8$ targets and $3 / 8 / 12$ seconds for the pitch/yaw/combined experiments based on feedback from the pilot study. In order to keep the subject focused on getting trained instead of being anxious about passing the tests, the time of the training session was not limited and the subject was not informed of the test details. In the study trials, the subject was asked to collect as many targets as possible within four minutes. All targets were placed randomly in an infinite $3 \mathrm{D} \mathrm{VE}$ and the distribution procedure was adjusted to generate the same level of challenge. After finishing each sub-experiment, the subject answered a post-questionnaire to indicate his/her favorite board mode and to rate both board modes comparatively regarding the intuitiveness, efficiency, precision, realism, ease of learning, ease of use, fatigue, after effects (e.g., loss of balance), fun, and sense of presence using a six point scale. The system recorded the $3 \mathrm{D}$ position and orientation of the virtual board and the virtual camera, as well as the user's performance, i.e., the number of targets collected and the time spent on passing the pretests. The whole user study took about one hour and fifteen minutes on average for each subject to complete.

The user study was approved by the institutional review board (IRB) and 30 undergraduate students from the social science department of Worcester Polytechnic Institute were recruited with a reward of elective course credits. Of the 24 subjects who successfully finished the study, 16 were males and 8 were females, 11 surfed using a goofy stance (right foot forward) and 13 by the regular stance (left foot forward). Their ages ranged from 18 to 27 years $($ mean $=20.7, S D=1.8)$, height from 154.9 to 195.6 centimeters $($ mean $=174.2, \mathrm{SD}=11.1)$, weight from 46.3 to 97.5 kilograms $($ mean $=73.3, \mathrm{SD}=13.1)$, and self-evaluated balance skill from 2 to 8 points $($ mean $=6.2, \mathrm{SD}=1.8)$. One subject reported board surfing in real life monthly, eight yearly, five once and 10 never. One subject played surfing-type video games yearly (Wii Sport), four once (Wii Sport and Tony Hawk) and 19 never. Two subjects used the Wii Fit Balance Board yearly, three once and 19 never. And finally, two subjects reported using a practice board in the fitness center monthly, two once and 20 never.

\subsection{Hypotheses}

Compared to the IBB, the ETB provides more realistic equilibrioceptive feedback but also requires additional body movement to maintain balance. Therefore we hypothesize that subjects will find the ETB to be subjectively preferable, but 
objectively less efficient than the IBB for the travel tasks. In detail, our hypotheses are:

H1: More subjects will prefer using the ETB over the IBB in all sub-experiments.

H2: The subject ratings of the ETB regarding intuitiveness, realism, sense of presence, fun, fatigue, and after effects will be significantly higher than that of the IBB in all sub-experiments.

H3: The subject ratings of the IBB regarding efficiency, precision, ease of learning, and ease of use will be significantly higher than that of the ETB in all sub-experiments.

H4: Using the IBB, subjects will spend significantly less time on passing the pre-tests than with the ETB in all sub-experiments.

H5: The number of targets collected using the IBB will be significantly greater than with the ETB in all sub-experiments.

\section{Results}

\subsection{Qualitative Measures}

The average scores and p-values for the qualitative measures are shown in Table 3 with significant differences shown in bold. The rating scores from the three post-questionnaires were analyzed using two-sided Wilcoxon signed-rank test with a threshold of 0.05 for significance on all questions. For all three subexperiments the ETB was rated to be significantly more intuitive, realistic, fun, and to offer a higher level of presence than the IBB. In addition, the ETB was significantly more tiring in the pitch and yaw sub-experiments and created more severe after effects (e.g., loss of balance) in the pitch and combined sub-experiments. No significant differences were found for the other six questions.

Regarding the subjects' general preference, we found most users preferred using the ETB, 20 subjects $(83 \%)$ in the pitch and combined sub-experiments and 18 subjects $(75 \%)$ in yaw.

\subsection{Quantitative Measures}

The number of collected targets was analyzed using two-sided Wilcoxon signed-rank test with a threshold of 0.05 for significance. Since the standard to pass a pre-test was the same between the ETB and the IBB, in order to compare their ease of use, the time spent on passing the pre-tests was also statistically analyzed using single-factor ANOVA with a threshold of 0.05 for significance. As shown in Table 4, no significant difference was found between the two modes in any sub-experiment.

Half of the 24 subjects started with the IBB in all subexperiments while the other half started with the ETB. To examine learning effects, the performance data and the pre-test time between the first and the second used interface in each subexperiment were compared using the same approaches and the results are shown in Table 5. A significant learning effect (more targets collected, less pre-test time spent using the second interface) was only found in the pitch experiment.

Interested in how demographic factors such as age, gender, height, weight or previous surfing experience influence the subjects' performance, correlation analyses were conducted using the Pearson correlation coefficient between each of these factors and the performance data. No strong correlation was discovered between any of the pairs (all coefficients $<0.5$ ).

\subsection{Travel Path Visualization}

To get a better understanding of how the subjects used the surfboard interface, the travel paths in the pitch and yaw subexperiments were plotted. As shown in Figure 8, the black curve indicates the subject's travel path and the squares represent the targets he/she collected along the path. The colored triangles indicate the orientation of the subject's viewport in the virtual world, with samples near the beginning of the trial being absolute red and those near the end an absolute blue color. The green spline connects all collected targets and the red-cross icons represent uncollected targets.

Table 3. The result of the qualitative data analysis

\begin{tabular}{|c|c|c|c|c|c|c|}
\hline \multirow{2}{*}{ Question } & \multicolumn{2}{|c|}{ Pitch } & \multicolumn{2}{|c|}{ Yaw } & \multicolumn{2}{|c|}{ Combined } \\
\hline & ETB & IBB & ETB & IBB & ETB & IBB \\
\hline \multirow{2}{*}{ Intuitiveness } & 4.6 & 3.6 & 3.9 & 3.4 & 4.3 & 3.4 \\
\hline & \multicolumn{2}{|c|}{$p=0.009$} & \multicolumn{2}{|c|}{0.038} & \multicolumn{2}{|c|}{0.002} \\
\hline \multirow{2}{*}{ Efficiency } & 4.5 & 4.2 & 3.1 & 3.1 & 3.1 & 3.0 \\
\hline & \multicolumn{2}{|c|}{0.253} & \multicolumn{2}{|c|}{0.902} & \multicolumn{2}{|c|}{0.519} \\
\hline \multirow{2}{*}{ Precision } & 4.1 & 4.0 & 3.1 & 3.0 & 3.3 & 3.2 \\
\hline & \multicolumn{2}{|c|}{0.826} & \multicolumn{2}{|c|}{0.557} & \multicolumn{2}{|c|}{0.861} \\
\hline \multirow{2}{*}{$\begin{array}{c}\text { Ease of } \\
\text { Learning }\end{array}$} & 4.7 & 4.7 & 3.7 & 4.0 & 3.2 & 3.5 \\
\hline & \multicolumn{2}{|c|}{0.681} & \multicolumn{2}{|c|}{0.098} & \multicolumn{2}{|c|}{0.131} \\
\hline \multirow{2}{*}{ Ease of Use } & 4.8 & 4.7 & 3.5 & 3.8 & 3.4 & 3.3 \\
\hline & \multicolumn{2}{|c|}{0.786} & \multicolumn{2}{|c|}{0.096} & \multicolumn{2}{|c|}{0.617} \\
\hline \multirow{2}{*}{ Realism } & 4.7 & 3.1 & 4.0 & 3.2 & 4.4 & 3.3 \\
\hline & \multicolumn{2}{|c|}{$<0.001$} & \multicolumn{2}{|c|}{0.002} & \multicolumn{2}{|c|}{$<0.001$} \\
\hline \multirow{2}{*}{ Presence } & 4.5 & 3.6 & 4.1 & 3.4 & 4.4 & 3.5 \\
\hline & \multicolumn{2}{|c|}{$<0.001$} & \multicolumn{2}{|c|}{$<0.001$} & \multicolumn{2}{|c|}{$<0.001$} \\
\hline \multirow{2}{*}{ Fatigue } & 2.8 & 2.3 & 3.3 & 2.8 & 3.1 & 2.8 \\
\hline & \multicolumn{2}{|c|}{0.036} & \multicolumn{2}{|c|}{0.028} & & \\
\hline After Fffect & 1.5 & 1.3 & 1.8 & 1.5 & 1.8 & 1.6 \\
\hline ATter Det & & & & & & \\
\hline Fun & 5.0 & 4.1 & 4.3 & 3.6 & 4.7 & 3.8 \\
\hline & & & & & & \\
\hline
\end{tabular}

Table 4. The result of the performance data analysis

\begin{tabular}{|c|c|c|c|c|c|c|}
\hline \multirow{2}{*}{ Exp } & \multicolumn{2}{|c|}{ Pitch } & \multicolumn{2}{c|}{ Yaw } & \multicolumn{2}{c|}{ Combined } \\
\cline { 2 - 7 } Performance & ETB & IBB & ETB & IBB & ETB & IBB \\
\hline $\begin{array}{c}\text { Number of } \\
\text { targets }\end{array}$ & 87.2 & 79.3 & 29.6 & 29.9 & 15.9 & 16 \\
\cline { 2 - 7 } & \multicolumn{2}{|c|}{$p=0.076$} & \multicolumn{2}{|c|}{$p=0.562$} & \multicolumn{2}{|c|}{$p=0.446$} \\
\hline Time to pass & 72.1 & 76.6 & 120.9 & 118.1 & 147.6 & 176.9 \\
\cline { 2 - 6 } the pre-test & \multicolumn{2}{|c|}{$\begin{array}{c}F(1,46)=0.86, \\
p=0.359\end{array}$} & \multicolumn{2}{c|}{$\begin{array}{c}F(1,46)=0.26, \\
p=0.615\end{array}$} & \multicolumn{2}{c}{$\begin{array}{c}F(1,46)=1.00, \\
p=0.322\end{array}$} \\
\hline
\end{tabular}

Table 5. The result of the learning effect analysis

\begin{tabular}{|c|c|c|c|c|c|c|}
\hline \multirow{2}{*}{$\begin{array}{r}\text { Exp } \\
\text { Performance }\end{array}$} & \multicolumn{2}{|c|}{ Pitch } & \multicolumn{2}{|c|}{ Yaw } & \multicolumn{2}{|c|}{ Combined } \\
\hline & 1st & 2nd & 1st & 2nd & 1st & 2nd \\
\hline \multirow{2}{*}{$\begin{array}{l}\text { Number of } \\
\text { targets }\end{array}$} & 77.8 & 88.7 & 29.6 & 31.1 & 16.3 & 16.3 \\
\hline & \multicolumn{2}{|c|}{$p=0.003$} & \multicolumn{2}{|c|}{$p=0.322$} & \multicolumn{2}{|c|}{$p=0.988$} \\
\hline \multirow{2}{*}{$\begin{array}{l}\text { Time to pass } \\
\text { the pre-test }\end{array}$} & 79.9 & 68.8 & 133.1 & 110.9 & 175.0 & 155.6 \\
\hline & \multicolumn{2}{|c|}{$\begin{array}{c}F(1,46)=5.67, \\
p=0.021\end{array}$} & \multicolumn{2}{|c|}{$\begin{array}{c}F(1,46)=2.09 \\
p=0.152\end{array}$} & \multicolumn{2}{|c|}{$\begin{array}{c}F(1,46)=0.69, \\
p=0.413\end{array}$} \\
\hline
\end{tabular}

Although the subjects were informed of the endless VE and the infinite targets, looking through the travel paths we found that many subjects travelled slowly and within a local area as if the number of targets was limited. To check how the travel speed related to the number of collected targets, a correlation analysis was done using the Pearson correlation coefficient. A strong correlation was found for the pitch sub-experiment of using both the ETB $(\mathrm{R}=0.847)$ and the IBB $(\mathrm{R}=0.744)$, meaning the faster the subjects travelled, the more targets they collected. However, no correlation was found for the yaw $(\mathrm{R}=0.309$ and 0.425 for ETB and IBB, respectively) and the combined experiment $(\mathrm{R}=$ - 
0.124 and -0.165$)$, indicating a successful subject can either travel at a high speed to collect from the greater number of available targets or travel slowly and carefully to collect every target close to him/her. The 24 subjects were categorized into six groups empirically with regard to the distribution of their surfing speed and number of collected targets. The result is shown in Table 6. "P", "Y", and "C" refer to the pitch, yaw, and combined subexperiments, respectively.

Table 6. The 24 subjects categorized into groups based on the distribution of their performance and travel speed

\begin{tabular}{|c|c|c|c|c|c|c|c|c|c|}
\hline Perf. & \multicolumn{3}{|c|}{ Low } & \multicolumn{3}{c|}{ Medium } & \multicolumn{3}{c|}{ High } \\
\hline Speed & $\begin{array}{c}2 \\
(\mathrm{P})\end{array}$ & $\begin{array}{c}0 \\
(\mathrm{Y})\end{array}$ & $\begin{array}{c}1 \\
(\mathrm{C})\end{array}$ & $\begin{array}{c}1 \\
(\mathrm{P})\end{array}$ & $\begin{array}{c}1 \\
(\mathrm{Y})\end{array}$ & $\begin{array}{c}0 \\
(\mathrm{C})\end{array}$ & $\begin{array}{c}0 \\
(\mathrm{Y})\end{array}$ & $\begin{array}{c}2 \\
(\mathrm{C})\end{array}$ \\
\hline Normal & 0 & 5 & 4 & 8 & 4 & 6 & 4 & 1 & 4 \\
$(\mathrm{P})$ & $(\mathrm{Y})$ & $(\mathrm{C})$ & $(\mathrm{P})$ & $(\mathrm{Y})$ & $(\mathrm{C})$ & $(\mathrm{P})$ & $(\mathrm{Y})$ & $(\mathrm{C})$ \\
\hline Fast & 0 & 2 & 1 & 1 & 5 & 5 & 8 & 6 & 0 \\
$(\mathrm{P})$ & $(\mathrm{Y})$ & $(\mathrm{C})$ & $(\mathrm{P})$ & $(\mathrm{Y})$ & $(\mathrm{C})$ & $(\mathrm{P})$ & $(\mathrm{Y})$ & $(\mathrm{C})$ \\
\hline
\end{tabular}

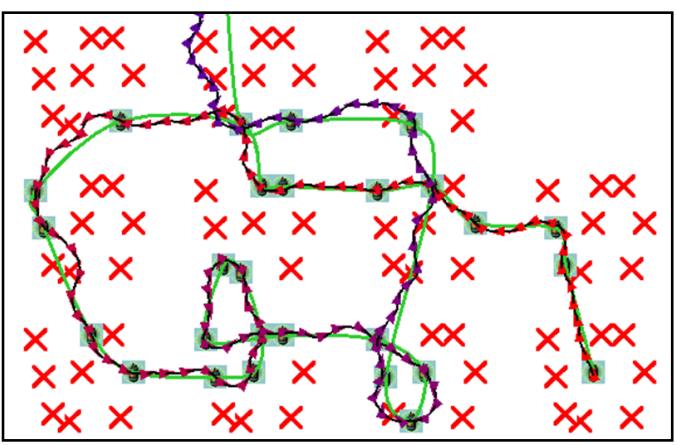

Figure 8 . The subject avoided taking sharp turns to get the most targets $(210 \mathrm{~m} / \mathrm{s}$, collected 48 targets in four minutes)

Inspecting Table 6, we found that although there is no strong correlation between speed and performance in the yaw experiment, most subjects achieved higher performance by surfing faster (six subjects in the fast-high group, one in the normal-high, and none in the slow-high group). From the travel paths of these subjects (such as in Figure 8) we found that they not only travelled faster for more available targets but also avoided taking sharp turns on their paths. On the other hand, for the combined trials, Table 6 revealed that subjects achieved better performance by not surfing too fast (two subjects in the slow-high group, four in the normal-high group, and none in the fast-high group).

\section{Discussion}

The qualitative results supported $\mathbf{H 1}$ and $\mathbf{H 2}$, that most users preferred the ETB in general because it is more intuitive, realistic, fun, and leads to a higher level of presence, despite the higher fatigue and after effects (e.g., loss of balance) resulting from it. However, against $\mathbf{H 3}, \mathbf{H 4}$ and $\mathbf{H 5}$, the additional physical effort to stay balanced on the tilt platform did not degrade the efficiency and precision, according to both the qualitative and quantitative results. This unexpected result is nevertheless similar to Zhai's results of comparing isometric and elastic hand-held trackball devices for 3D object manipulation tasks [26] with the exception that the performance superiority of the ETB was not found in the early pre-test stages. There can be many explanations. For example, it is possible that the intuitiveness, realism and fun of operating the ETB due to its richer equilibrioceptive feedback stimulated the subjects to perform well, but the extra motion effort to tilt it (up to 10 degrees in every direction) counterbalanced these benefits. Although it is uncertain whether the performance of the ETB will degrade significantly as the scale of tilt increases, we strongly feel it is necessary for future interface designers to incorporate more motion in their designs, thereby providing users with more proprioceptive, equilibrioceptive, or vestibular feedback to make the interaction more intuitive, immersive, and enjoyable.

The lack of learning effects may be due to the complexity of the task as well as the effect of the pre-tests which were targeted at ensuring the same basic skill level for all conditions. We believe that the subjects reached the same level of basic skill after passing the pre-tests for both interfaces. Asymmetric learning effects have been a common threat to within-subjects experiment designs, therefore we would like to suggest this pre-test design because it strives to eliminate this asymmetry by balancing the skill transfer between trials.

The subjects' ages, heights, weights, and balance skills did not strongly correlate to their performance probably because the calibration process normalized their ability to tilt or balance the board to the same level. Their surfing experiences in real life and video games did not correlate with their performance either, which may indicate the surfboard metaphor was more of an abstraction instead of a simulation of the 2D surfing scenario in real life. One may argue that the control law of our surfboard interface did not strictly follow the real world physical rules governing the motion of skateboard or snowboard. Nonetheless, the subjects felt this simplified design was very easy to understand, learn, and use. It will be interesting and necessary to compare the surfboard to the Segway simulator [21] and the Joyman interface [10], which more strictly followed complex physical laws, to see how much a travel interface could be abstracted from real life counterparts to achieve similar or even better performance and user experience.

Analysis of the travel paths revealed that the subjects performed better by traveling faster and avoiding taking sharp turns in the yaw sub-experiment. This is because the virtual board yaw was rate controlled, and in order to take sharp turns, the user had to slow down or stop, lean to the correct direction and then speed up, while on the other hand, because the virtual world repeated endlessly, he/she could instead ignore the missed targets and keep moving on to get more targets. Nevertheless, the subjects performed better at normal speed in the combined subexperiment. The reason may be that the integrated control of both pitch and yaw made the combined tasks more challenging and the subjects were not confident of controlling the interface at fast speeds. These discoveries indicate that the surfboard interface may not be as efficient when compared to pointing directed or gaze directed travel interfaces when frequent and sharp turns are necessary. Actually, we believe that most rate-controlled steering interfaces will not outperform position-controlled pointing interfaces for fast, sharp, and frequent direction changes. However, we believe that by turning slowly and gradually, the user will be better oriented, less nauseated, and able to form a better cognitive model of the $\mathrm{VE}$, as suggested by Bowman et al. [2] in their research of direct viewport transportation.

Lastly, as mentioned earlier, six of 30 subjects were not able to finish the study. Five of them dropped out because of cybersickness. The experimenter interviewed the subjects and three subjects blamed the IBB for causing their nausea. The reason, according to one of the subjects, was that "No matter how much the virtual board turns, the balance board always kept stationary, and this inconsistency between what my eyes see and what my body feels made me sick." This has not been an issue for the IBB in its typical, non-immersive video gaming usage. This is because when the user uses the IBB to travel through VEs rendered on the TV screen, his/her peripheral vision of the real world is in the same scale as his/her body movement. As a result, the 
inconsistency between the latter and the virtual world vision was more compromised than it is in an immersive system. Based on this argument, we again suggest future interface designers to incorporate more body motions in their design to avoid cybersickness induced by this real/virtual motion mismatch.

\section{CONCLUSION AND FUTURE WORK}

To summarize, we presented a novel leaning-based surfboard interface which can be used to travel in 3D VEs in two modes. A formal user study was conducted by comparing the two modes of performing 3D search and collect tasks to investigate the impact of equilibrioceptive feedback on user performance. The ETB was preferred by most users because of its intuitiveness, realism, fun, and sense of presence despite the fatigue and after effects involved. Given these benefits, the equivalent level of efficiency and precision, and the potential cyber-sickness induced by using the IBB, we suggested a general preference of elastic devices over isometric devices and several design guidelines to future $3 \mathrm{D} \mathrm{VE}$ travel interface designers.

The presented work will be extended in three directions. First, the surfboard interface will be compared to existing 3D travel interfaces such as joysticks as a baseline evaluation. Secondly, we will explore the factors that may cause cyber-sickness in the usage of leaning based interfaces for virtual world navigation tasks, and compare their effect in immersive (HMD) and non-immersive (TV screen) VR systems. Lastly, we are interested in the performance and cognition differences between different stances of using leaning-based travel interfaces, e.g., a comparison between our sideways-stance surfboard interface and the frontalstance Segway simulator [21].

\section{REFERENCES}

[1] S. Beckhaus, K. J. Blom, and M. Haringer. ChairIO - the ChairBased Interface. Ed. Magerkurth und Rotzler, Shaker Verlag, 2007.

[2] D. A. Bowman, D. Koller, and L. F. Hodges. Travel in Immersive Virtual Environments: An Evaluation of Viewpoint Motion Control Techniques, In Proceedings of IEEE Virtual Reality Conference'97, pp. 45-52, Albuquerque, NM, USA, 1997.

[3] D. A. Bowman, E. Kruijff, J. J. LaViola Jr., and I. Poupyrev. 3D User Interfaces: Theory and Practice. Addison-Wesley. 2005.

[4] G. Cirio, M. Marchal, T. Regia-Corte, and A. Lécuyer. The Magic Barrier Tape: a Novel Metaphor for Infinite Navigation in Virtual Worlds with a Restricted Walking Workspace. In Proceedings of the ACM Symposium on VRST'09, pp. 155-162, Kyoto, Japan, 2009.

[5] G. de Haan, E. J. Griffith, and F. H. Post. Using the Wii Balance Board $^{\mathrm{TM}}$ as a Low-Cost VR Interaction Device. In Proceedings of the ACM Symposium on VRST'08, pp. 289-290, New York, NY, USA, 2008.

[6] H. Iwata, H. Yano, H. Fukushima, and H. Noma. CirculaFloor: A Locomotion Interface Using Circulation of Movable Tiles. In Proceedings of IEEE Virtual Reality Conference'05, pp. 223-230, Bonn, Germany, 2005.

[7] H. Iwata, H. Yano, and F. Nakaizumi. Gait master: A Versatile Locomotion Interface for Uneven Virtual Terrain. In Proceedings of IEEE Virtual Reality Conference'01, pp. 131-137, Yokohama, Japan, 2001.

[8] H. Iwata and Y. Yoshida. Path Reproduction Tests Using a Torus Treadmill. Presence: Tele-operators and Virtual Environments, vol. 8(6), pp. 587-597, 1999.

[9] J. J. LaViola Jr., D. A. Feliz, D. F. Keefe, and R. C. Zeleznik. Hands-Free Multi-Scale Navigation in Virtual Environments. In Proceedings of the Symposium on Interactive 3D Graphics'01, pp. 915, New York, NY, USA, 2001

[10] M. Marchal, J. Pettré, and A. Lécuyer. Joyman: a Human-Scale Joystick for Navigating in Virtual Worlds. In IEEE Symposium on $3 D$ User Interfaces'11, pp. 19-26, Singapore, 2011.
[11] M. R. Mine, F. P. Brooks Jr., C. H. Sequin. Moving Objects in Space: Exploiting Proprioception in Virtual-Environment Interaction. In Proceedings of ACM SIGGRAPH'97, pp. 19-26, Los Angeles, CA, USA, 1997.

[12] T. C. Peck, H. Fuchs, and M. C. Whitton. Improved Redirection with Distractors: A Large-Scale-Real-Walking Locomotion Interface and its Effect on Navigation in Virtual Environments. In Proceedings of IEEE Virtual Reality Conference'10, pp. 35-38, Waltham, MA, USA, 2010

[13] I. Poupyrev, M. Billinghurst, S. Weghorst, and T. Ichikawa. The GoGo Interaction Technique: Non-linear Mapping for Direct Manipulation in VR. In Proceedings of ACM UIST'96, 1996.

[14] S. Razzaque, Z. Kohn, and M. C. Whitton, Redirected Walking. In Proceedings of Eurographics' 01, Manchester, UK, 2001.

[15] B. Riecke, B. Bodenheimer, T. McNamara, B. Williams, P. Peng, and D. Feuereissen. Do We Need to Walk for Effective Virtual Reality Navigation? Physical Rotations Alone May Suffice. In Proceeding of Spatial Cognitions'10, pp. 234-247, 2010.

[16] E. A. Suma, S. L. Finkelstein, M. Reid, A. Ulinski, and L. F. Hodges. Real Walking Increases Simulator Sickness in Navigationally Complex Virtual Environments. In Proceedings of IEEE Virtual Reality Conference'09, pp. 245-246, Lafayette, LA, USA, 2009.

[17] E. A. Suma, D. M. Krum, S. Finkelstein, and M. Bolas. Effects of Redirection on Spatial Orientation in Real and Virtual Environments. In Proceedings of IEEE Symposium on 3D User Interfaces'11, pp. 35-38, Singapore, 2011.

[18] R. M. Taylor, T. C. Hudson, A. Seeger, H. Weber, J. Juliano, and A. T. Helser. VRPN: A Device-Independent, Network-Transparent VR Peripheral System. In Proceedings of the ACM Symposium on VRST'01, Banff, Canada, 2001.

[19] J. N. Templeman, P. S. Denbrook, and L. E. Sibert. Virtual Locomotion: Walking in Place through Virtual Environments, Presence: Tele-operators and Virtual Environments, pp. 598-617, 1999.

[20] M. Usoh, K. Arthur, M. C. Whitton, R. Bastos, A. Steed, M. Slater, and F. P. Brooks Jr., Walking > Walking-in-Place > Flying, in Virtual Environments. In Proceedings of ACM SIGGRAPH '99, Los Angeles, CA, USA, 1999.

[21] D. Valkov, F. Steinicke, G. Bruder, and K. H. Hinrichs, Traveling in 3D Virtual Environments with Foot Gestures and a Multi-Touch enabled WIM. In Proceedings of Virtual Reality International Conference'10, pp. 171-180, 2010.

[22] M. Vidal, M. Amorim, and A. Berthoz, Navigating in a Virtual Three-dimensional Maze: How do Egocentric and Allocentric Reference Frames Interact? Cognitive Brain Research 19(3), pp. 244-258, 2004.

[23] J. Wang and R. W. Lindeman, Isometric versus Elastic Surfboard Interfaces for Locomotion in Virtual Reality. In Proceedings of the IASTED Conference'11, Washington D.C., USA, 2011

[24] C. A. Zanbaka, B. C. Lok, S. V. Babu, A. C. Ulinski, and L. F. Hodges. Comparison of Path Visualizations and Cognitive Measures Relative to Travel Techniques in a Virtual Environment. IEEE Transactions on Visualization and Computer Graphics, vol. 11(6), pp. 694-705, 2005.

[25] S. Zhai, Human Performance in Six Degree of Freedom Input Control, Ph.D. Thesis, University of Toronto, 1995.

[26] S. Zhai, Investigation of Feel for 6DOF Inputs: Isometric and Elastic Rate Control for Manipulation in 3D Environments. In Proceedings of the Human Factors and Ergonomics Society 37th Annual Meeting'93, pp. 323-327, Seattle, WA, USA, 1993.

[27] S. Zhai and P. Milgram, Human Performance Evaluation of Manipulation Schemes in Virtual Environments. In Proceedings of IEEE Virtual Reality Conference'93, pp. 155-161, Seattle, WA, USA, 1993. 